DOI: $\underline{\text { https://doi.org/10.24297/iap.v16i1.8485 }}$

\title{
The Algorithm of Information and the Origin of Basic Particles
}

\author{
Bhekuzulu Khumalo \\ 305 - 19 Northern Heights Drive, Richmondhill, Ontario, Canada \\ bhekuzulu.khumalo@gmail.com
}

\begin{abstract}
Everything is information because everything will inform you about itself through our senses, there is no other way to say it when discussing the philosophy of science. All information has similar basic characteristics including randomness. The reality of randomness gave the concepts to the theory of the algorithm of information the basis of all degrees of randomness. Everything is about relationships; the algorithm of information is the tool existence uses to determine the chances of relationships. If everything is about relationships, then relationships must begin with the particles. Not the basic particles having relationships with other basic particles as it was first postulated. The basic particle itself must be the result of an event and thus a relationship, it was not always there. This paper aims to shed some light on the question of where do particles come from? What relationships led to the event that created elementary particles?
\end{abstract}

Keywords: Algorithm of Information, Energy, Origin of Mass, Mass, Massless, Origin of Particles, Particles, Theory of Everything

Aims: The aim of this paper is to take the ideas that everything is information and all information packages have the same basic characteristics, these being:

- discrete

- independent

- can be isolated

- can be used to compute

- $\quad$ consistent (why it is important to understand logic)

- random

- $\quad$ Everything is in a Relationship

We use the word information rather than energy, why? Nobody has really got energy quite correctly or told us what precisely it is. But information is something that informs us. If we say everything is energy, its kinetic energy, potential energy, the ability to do work, well-saying everything has ability to do work, would be also correct, but information is more precise because we are not defining everything for a rock, but for intelligent life. Knowledge is for us, and we cannot separate ourselves from the universe, information means we are informed by the universe what it is.

Having these similar characteristics one can accept the thought that there must be laws of existence or law of existence that cannot be disobeyed by anything in existence, they affect everything. This means the study of everything began on the wrong footing. The idea of a theory of everything started as an argument in physics involving the likes of Einstein, a single equation applying to everything, a single principle applying to everything. 
What if there are many principles, or at the least more than one principle that applies to everything, then the study of everything must be itself a discipline.

We must understand what everything means. If you do not know the meaning of the word, one should not use it, use another word. Everything means from dictionary.com "every single thing or every particular of an aggregate or total; all." Using a big word like everything one must be sure to include everything, there can be no excuses.

Being a discipline unto itself, the theory of everything, it means it can be categorized/ classified as a discipline. The first of these laws recognize that everything is about the relationship and that every relationship leads to a loss of freedom. As it is about relationships, these relationships cost energy to maintain and hence the loss of freedom. Every relationship is a random event, what does this mean concerning the origin of particles, this paper aims to delve into this question. When talking of categorization, it was a certain 1Suzzana Ngatai who pointed out the natural process that leads to categorization. Important to appreciate the whole process. Because categorizations humans make are based on characteristics of information, it follows that information self categorizes and humans are merely mimicking nature, all other information in their minds, we can see the algorithm giving out all these categorizations, a very important concept category of information.

As usual, a thought experiment will be provided for some aspects of the theory of the origin of particles, experiments are put your money where your mouth is in science. If one feels comfortable with their understandings of relationships and that everything is information one can immediately go to section 6 about the algorithm of information.

\subsection{Everything is Information}

In simplistic terms everything is information because it will all inform you about itself, by merely looking at a flower, we know it is a flower, it has told us what it is. Information comes in packages, the smallest package being an elementary particle and the largest information package being a galaxy. In terms of size, every other information package exists, this includes, stars, humans, lions, cars, beaches, a grain of sand, oceans, a drop of water, asteroids, planets, gold, anything you can imagine is an information package.

To get to the idea that everything is information and the reality that everything behaves the same one needed to remove from their mind the original sin mentality of man, that one can look at the universe without human beings. Once one removes human beings at the onset of their investigation, they can never see how everything has similar basic characteristics because they are not including themselves in everything when clearly, they are there and thus for this moment in the universe's history need to be included.

It was a cultural outlook that stopped humans from getting to the idea that everything is information and thus leading to the concept of relationships that will be discussed later. This idea that somehow mankind was born evil and thus is removed from the picture there will be a better world, this sipped into the minds of physicists who decided to look for theories explaining everything but removing humanity.

That everything has the same basic characteristics should not have been something realized in the 21st century, it belongs in the 1950's - 1960's era of physics, the thought patterns involved. The results, the algorithm of information, however, does belong in the 21st century and could not be arrived at without understanding that everything is in a relationship that comes from everything has similar basic characteristics because it is all information.

The reason why everything has the same basic similar characteristics is that it is all made up of the same basic materials, the elementary or basic particles. Just as all say plastic products have similar characteristics, or those things made from iron, at the least they all rust, everything has these basic characteristics because it is all made up of the same stuff that has those basic characteristics and passes those characteristics on to whatever is made from it. 
To appreciate the algorithm of information, of all the basic characteristics of information, that information thus all things in existence are discrete, independent, can be isolated, can be used to compute, consistent, and are random, randomness is the characteristic to appreciate. Every action or change in this universe is random, there is the chance that action or event does not take place. The positions of the stars is due to random events, a star could easily have been positioned somewhere else if events had been different, it might not even exist, just as one's existence is a random event, parents might never have met, and even if one plans to meet somebody at 3 pm they might meet up with an accident.

When talking of randomness, it means every event in this universe is determined and limited by a probability function. There are impossible scenarios that is why one must also include the word limit, there are limitations. One can jump higher and higher, but one will not jump over a building, there is a limit, but that limit can vary widely in type. A human without special equipment will never jump over a building, but we can have an infinite variety of visible light. You can mix paint in infinite variations. Thus, photon emitted can take on an infinite variety of colors, but no photon will create an event that will pass a 10-meter-thick wall of lead that probability function has clear limits. The algorithm of information is the basis of all randomness, this paper seeks the beginnings of randomness, the original function of randomness.

\subsection{Relationship}

Though each information package is discrete, independent, can be isolated, can be used to compute, is consistent, and is random, it is in a relationship and is itself created by relationships, that is why we can isolate information. We know something by isolating it, that is a duck, that is a mountain, that planet is Venus, we isolate it from the other information packages, but all the mentioned information packages belong to the information package that is our solar system, that belongs to the larger information package the Milky Way galaxy, that belongs to the larger information package the universe.

Relationships need energy to be maintained, need the energy to sustain themselves this energy comes from the information packages leading to the first law of information, the first law of everything. A law of everything must apply to everything. The logic of the law itself at this moment is over 7 decades old. It was missed because of a petty argument and petty minute details; all human beings can be petty. You cannot take the human being out of the picture, just that single first misstep will mean you can't get to a law of everything because the human being is part of everything. A particle just like a human being is part of relationships and is from a relationship, by the end of this paper one should appreciate the thought that a particle is from a relationship. And we know it is a particle because we have isolated it.

The 1 first law of information states that every relationship leads to a loss of freedom. This loss of freedom is due to the energy needed to maintain the relationship. One could also say that every relationship has a cost. However, cost supposes a gain and a loss, loss of freedom implies not as free as before the relationship, and that is a fact. Understanding that everything is in a relationship brings about what was fantastic could have been more fantastic. A black hole has been explained so well theoretically that artists could imagine it. When the EHT (Event Horizon Telescope) gave us their pictures of what is around a black hole, it was not overwhelming, it was familiar with artists' interpretations of the theories about a black hole. An accurate picture of a black hole comes from the information we already have, the temperature. Merely by understanding the relationships around temperature and a black hole a true picture could have been taken, beyond artist imagination.

When one looks at it from a wholly human accepted view of the philosophy of today, every relationship might very well have a cost to humans. Everything is about gain and loss, a characteristic common to humans and many other forms of life, with the human having the most sophisticated brain. But this wording does not apply to everything. That proton in a human being does not feel less or more of a gain than being in gold, in a star, in feces, but that proton by events that make it be part of a human means it is less free as it can't be in a star, gold, or feces. The probability function adjusts or collapses after the event and new functions come to be. 
Why the talk of functions? Because every information package comes from random events, and it is at a random location. When conditions change probability of events changes. This applies to basic particles, galaxies, humans, economics, chemistry, trees, zebras, planets, everything. When heat is added to water, the chances of water boiling increase, the probability function that determines likelihood of boiling changes indicating greater chances of boiling, there might not be enough fuel to get the water to boil even though heat has been added.

The likelihood of things happening is always changing. Take a fruit tree, when the fruit ripens, it falls off the tree. But there are many ways it can fall of a tree, a bird picking at it be it ripe or not. Thus, from the moment the fruit is there, there is a chance that it will fall off the tree. Therefore, every day, the probability function of the fruit falling off the tree is adjusted, and when the fruit falls off the tree that probability function collapses, and other functions take over as that relationship of the fruit going back and being stuck to the tree is zero.

How much energy does a relationship take? The amount of energy a relationship takes is the amount of energy it takes to undo the relationship. For example, water is made up of hydrogen and oxygen, the hydrogen and oxygen are in a relationship. The amount of energy it takes to break up the relationship, to separate oxygen and hydrogen is the amount of energy it took to have the relationship. In the previous 2paper "A Treatise of Information, Knowledge, Economics, and Technology" the early stages that everything is about relationships and those relationships need energy to maintain. This energy used to maintain a relationship is called "Free Energy" because though in a relationship, that making up the relationship is not destroyed. That is why we can isolate information, that is why hydrogen can be separated from oxygen, and the hydrogen and oxygen is not something else after the relationship.

The reality of complexity must be understood in order to grasp the probability of a relationship and the energy used for the relationship. Basic particles are the simplest things known, but it takes powerful particle colliders to break them up. It takes an ax and muscle power to bring down a complex information package like a tree. A frog is more complicated than a black hole, thus, in reality, it is easier to understand a black hole than a frog. The relationships involved in creating a frog are more complex than those involved in creating a black hole. Thus, anything around a black hole cannot be more important than understanding a frog, there is more to learn from a frog. A black hole is far away, it's fascinating, mysterious, frogs are noisy pests. Complexity does not render importance, any piece of information missing would mean a different universe. If you take out humans, why not take out frogs, why not black holes, they are all just packages of information with the same basic characteristics.

The more complex something, the easier it is to destroy, because the amount of energy holding the relationships gets weaker and weaker. Thus, it is easier to destroy a frog than it is to destroy a black hole. Life is meant to be, otherwise it would not be, otherwise the very laws of information would be different, there might be no gravity, electrons, we just cannot speculate, because reality is humans are here, a universe without humans at this moment is a universe with different laws from this one.

Modern technology through the colliders has given us greater insight into relationships and the way these probability functions work. To destroy a rock takes a sledgehammer. To destroy the molecules and get pure elements takes more energy, fire, iron ore to iron, or the act of separating molecules, crude oil into its many constituencies. To destroy the elements takes nuclear explosions, to destroy the parts of an atom, the basic particle takes more energy, a collider. It is difficult to destroy elements; they are more complex than basic particles but still difficult to destroy. But a human, a complex creation at most, exists for just over 100 years, and then due to its complexities, things break down.

As it takes less and less energy to physically isolate information, the more complex information becomes, it means the probability function for amount of energy to destroy a relationship takes less and less energy and thus changes each time a piece of information enters a relationship. The more relationships, the easier it is to separate the relationships. A marriage though a relationship will not last as long as oxygen, which is also a result of relationships. 
Probability functions depend on many factors, and thus the events that created that relationship. One of those factors that determine the predictability of an event of physical isolation is how much energy is needed to break up the relationship. The more complex, the less energy is needed to maintain that relationship.

\subsection{How Did We Get Here?}

Where is the concept of everything from, information from such that the logic leads us to an irrefutable reality that everything is in a relationship, and the first law of everything "every relationship leads to a loss of freedom". How come it was missed for a very long time when the idea of something that explains everything has been around for some time.

It comes down to philosophy even though some scientists kid themselves that philosophical concepts have no meaning. The question of life is central to philosophy. Take questions people ask themselves, a soldier being the only survivor, why am I alive? Does a tree make a noise when nobody hears? What if it went this way? These are all intellectual questions and might sound silly, to ask oneself why they are existing, the answer is simply because they are existing at its most fundamentals without any qualifications.

Though life is a random event as the location of any piece of information is in relationships, it is still information and exists. A human is a random event and spends its life not only created by random events but influenced by random events throughout their lifetime. Humanity is no more a random event than a frog, a fish, a star or an asteroid. Not forgetting that information is all made of the same stuff with unique relationships leading to different characteristics and roles. Einstein asked a question is life important in this universe, can we get a theory of everything that has nothing to do with life. He once asked what does a mouse by merely looking at the moon change the nature of the universe. Yes, it does, the location of photonic energy changes, the eyes of the mouse absorb photons that otherwise could have hit the back of the mouse or the ground. That photonic energy created a relationship in the eyes of the mouse not somewhere else. Events that otherwise would not have occurred, have occurred.

Another answer to answering the question is a human important to the universe can only be understood by not being fixated at life, just accept life and only then can one properly see things as they are leading straight to everything is in a relationship and that leads to a loss of freedom because relationships cost energy to maintain. This is common to everything in existence known and unknown. Whatever discovery is made, we understand it by that thing, telling us what it is, if it is an invention, tells us what it is when humans manipulate information and create relationships that otherwise would not have happened if humans did not exist, a frog will not invent a television. But that relationship is just manipulating relationships of information by human beings that are also just information.

Thus, because humans are just information like everything else, if one wants to take themselves out of the picture when looking at the universe, then wonder why they can't get to principles that explain everything. When we study a star, we are in a relationship with those stars. The relationship is that the light they emit reaches us and we can understand their behavior because of the energy patterns we receive from them. Those patterns of energy are the star telling us what it is, the more we look and understand, the more the star is telling us its various characteristics. If we decide to take out a human being an information package why not take out the stars in this mythical quest. Why not take a frog or a planet out of the equation, they are no less or more information packages like a human being?

As all information is discrete and can be isolated, if it was not discrete it would be impossible to isolate information. That discrete information that is being isolated is being isolated from a relationship, and we categorize it. By merely looking we are asking what that is, we are isolating information, we can do this because with sight photons they radiate tell us what they are, and we can avoid obstacles. We are always asking, being familiar with something allows us to get the answer almost instantaneously, we call this knowledge instantaneous knowledge, but if we are alive, we are asking every second, and universe is answering every second. We see, feel, hear, smell continuously, always asking and always receiving an answer. 
That everything is in a relationship, and the first law of information comes from a combination of knowledge economics and quantum mechanics, that is how the question is life important was answered. Knowledge economics gave us the properties of knowledge. Knowledge follows a property of consistency. This property of consistency is also followed by the most basic particles, a photon is a photon and can't be an electron at the same time. But then a star is a star and can't be an asteroid at the same time. Logic is built upon this principle. Knowledge comes in packages just as particles come in discrete packages. It important to understand that knowledge comes in packages. The idea that information comes in packages, that a star is an information package just as a human being, a frog, an asteroid, a galaxy, a black hole is from the idea that knowledge comes in packages. That is a significant contribution of knowledge economics to fully comprehending how everything works and behaves in the universe.

Once understanding that knowledge comes in packages and that the knowledge package can be as big as possible and as small as possible. But this knowledge we know is the reality out there, meaning that the reality out there from the smallest particle to the largest galaxy behaves in the same way as knowledge or we could never know it. It is quantum mechanics that tells us that the reality informs us what it is, it is all information because any information package can be used to compute. Though knowledge economics understood the principles of randomness, there are different routes to the same knowledge, quantum mechanics through experimentation proved the randomness of basic particles and the randomness of the universe from the most basic particle to the largest galaxy. Everything is determined by probability functions from each moment to each moment.

Knowledge economics is about the study humanity's most basic tool, the resource of the mind, knowledge. The mind belongs to a human being, it is about specifically human life, not all life, but it is about life, nonetheless. The thing that knowledge is, is what we have been informed by the universe, we can only know information packages from existence, if it does not exist it cannot be known. To understand that everything is in a relationship took a combination of understanding a resource used by life to exist, knowledge, and the look into the most basic of building blocks we know, the subatomic world. The resource of life has same basic characteristics as the universe. Even time is relative when it comes to knowledge just as it is relative with the rest of the universe.

We know what we know because we isolate things and say this is this. We are isolating stuff, and then we categorize it to suit ourselves. When we isolate this stuff, it is always telling us what it is its unique characteristics differentiating it from all the other stuff that is made up of the same thing just differentiated by relationships of information packages and thus same basic characteristics.

This idea of knowledge packages, as described in the paper "Point X and the Economics of Knowledge." Though our understanding of information has far exceeded that time of 2007, it was a paper establishing how knowledge comes in packages, that understanding with idea that everything is information leads to us understanding the first law. These packages of information come with the characteristics described above under the first section of this paper, the aims of the paper. That knowledge comes in discrete packages leads to the understanding that everything and anything can be used to compute, can be isolated, and it can only be isolated from a relationship. This leads to us being able to talk about the origins of particles, as usual, a good paper must propose an experiment, and a suitable experiment will be provided to prove the logic and theory of the origin of particles.

\subsection{Theory of Everything is a discipline}

As everything is information and there are certain characteristics that apply to everything, starting with the principle of consistency, a thing cannot be something else at the same time. This ensures that mathematics works and can be used to describe the phenomenon. It ensures we have a means $f$ identifying things. The law of consistency binds categories, we categorize things according to consistencies, patterns we identify outside the major basic characteristics. But it is the major characteristics that allow us to reach characteristics that are particular to a category. 
When things are categorized, and something is being investigated, one of the first questions that should be asked is why should this thing be different from all other phenomena in its category? The next question is how it is different. Given that things can be categorized, for example a general categorization is all information is discrete, if somebody says something is continuous, they must tell us why it is not discrete. It can't be up to somebody to prove something is not continuous, the burden of proof must rest on those who say something is continuous, defying all other information. All information is discrete, that means whatever you are investigating is discrete. It means gravity must be discrete. If everything else is discrete, it is not up to everybody else to prove that gravity is discrete, it is for those who say it is not discrete who must prove the impossible.

The same with magnets, if they are not discrete, it is up to those who say magnets defy everything else by giving impossible proof and leading people astray. Merely thinking about magnets as discrete makes it more exciting, the implications. Because there are north and south pole, and we know everything is limited by a probability function, being discrete, being a particle would mean magnetic particles are created in pairs, the pairs are entangled such that if one is north, we know without even measuring the other is south. When one particle changes to be south, the other automatically changes to north. The Donson, the discrete magnetic particle should be there.

Instead of being stuck on debates is something discrete, the question is why it should not be discrete, why should it break with consistency. Thus, quantum tunneling could never have been particles going back and forth in time to borrow energy though it was discussed in serious scientific circles. Of course, knowing the consistency of the universe, it has to do with relationships, and every relationship costs energy. Whilst mainstream where entertaining time travel to explain quantum tunneling those who understood everything is information was talking about time travel, they were talking about the 5 characteristics of the particles.

When we discuss the origins of particles it will be understood why time travel can at most be considered a funny concept, if it is to be real it proves will not come from the behavior of particles, it will have to be a concept created by the mind because it will be understood that the particles cannot do that. The image of a black hole. Photos are taken due to photons, heat is a photon, thus, to take a photo of a black hole it must be a mapping of heat, there is not much other information it is giving out. There is no photo of a black hole until there is consistency in desire with reality.

What does it take for anything to become a discipline or a sub-discipline? It takes a subject to have its own rules. As everything is information characteristics of information affect everything. These characteristics have been mentioned several times including it can be used to compute. Therefore, information is limited and thus can be categorized by characteristics. Secondly a discipline must have laws that define that discipline. The first law of information has been mentioned above, and it states that every relationship leads to a loss of freedom. This loss of freedom is due to the energy that is used to maintain that relationship. It is a law of everything, nothing that exists within our existence can break this law, everything is in a relationship and everything has lost freedom. This law cannot be broken scientifically or philosophically.

As the first law of information is about everything, this means that it crosses all disciplines and trades, whatever we do, whatever profession, whatever discipline is being studied, we are all dealing with relationships. Be we a chemist, economist, zoologist, sociologist, priest, boxer, quantum mechanics, accountant, nurse, astrophysicist, gardener, cook, carpenter, name it, you are dealing with relationships thus all disciplines are united, study the same phenomenon but different aspects of this phenomenon, information. The probability functions around these disciplines have to do with the energy exchange of relationships, quantity, and quality.

The information has a second law, a law that is about information must by its nature affect everything. The second law of information is inspired by a thought from Isaac Newton. The 6second law of information states "an information package will remain in its present state unless a relationship is established." This should not be difficult to understand. Iron ore will remain iron ore unless new relationships are established, and we end up with pure iron from smelters. Hydrogen will remain as it is until oxygen is introduced into the location and there is enough energy to unite the two elements to create water, the earth will rotate around the sun until energy is 
introduced that will either make the earth go closer to the sun or lose its orbit. The earth will rotate around the sun until another relationship changes that.

The mistake with the theory of everything seems to be that people were looking for one principle that affects all, what if there is more than one principle. All the principles will, of course, have to do with relationships that affect all. The first law of everything is about everything being in a relationship, and that relationship has an energy cost. It describes the universe as it is, everything is in a relationship, these relationships all take energy to maintain. A proportion of energy must be used to maintain that relationship, the proportion is determined by a probability function. For example, when a photon is absorbed by an atom, it gives all its energy, the atom enough to contain the photonic energy, the atom is not giving as much energy as the photon for the relationship. Not everything gives the same proportion of energy. Another example is a black hole, a star and its solar system if it still has one as it passes the event horizon of a black hole, a star gives all of itself, the black hole gives up the gravity needed to contain the energy of the stars.

The second law also deals with relationships. The earth will orbit the sun until a new relationship is introduced, that new relationship takes energy to maintain. This energy must be enough to offset the old relationship. The amount of energy needed to change its relative location is again determined by a probability function. The theory of everything is a separate subdivision about laws and relationships that affect all information. Thus, it is wrong to look for a single magical relationship, there are many relationships just as you would find when studying a subdivision like thermodynamics. Though there is always energy being exchanged the amount of energy, the proportion of energy being exchanged to maintain a relationship depends on different factors, including complexity, gravity, vacuum, other relationships in the vicinity.

We know two laws that affect everything, more will be found. The laws that affect everything affect every discipline, that everything is in a relationship is not just about physics and behavior of say atoms and molecules, but also sociology, biology, psychiatry (our thinking is because of relationships we have and have had), geology, boxing, the probability functions will all have the same principles behind them.

\subsection{The Algorithm of Information}

Algorithm of information introduced in the paper Knowledge Economics: Summary and rationality. To quote from the paper, "...argument around rationality would be impossible without the algorithm of information, as it shows that for every question, the answer is a probability. Knowledge economics follows the principles of the algorithm of information, thus linking it to all other disciplines as all other disciplines are dealing with information, and thus the principles of the algorithm of information can never be escaped."

The concept was introduced around economic decisions centered around the idea of knowledge economics. But we need to understand it now in a different manner such that we can get to the origins of particles. We need to understand its origins, thus getting to understanding origins before some of the greatest events in the history of the universe, greatness being the amount of information involved.

\subsection{Understanding the Algorithm of Information}

Take a piece of information, Ki. For Ki to be involved in a relationship there are options available and each option is determined and limited to by a probability function that is easily laid out in an algorithmic manner, that this can be done in this manner is important in understanding the origin of things. This concept of the chance is illustrated in figure 1. 


\section{Figure 1: Probability of a Relationship}

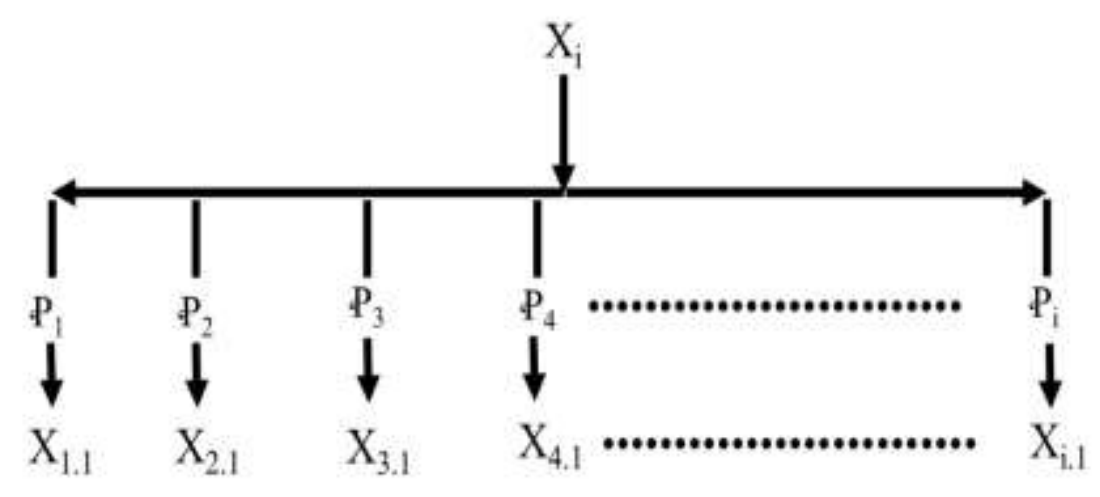

Given figure one the is probability $P_{1}$ that $X i$ will be in a relationship, and $X_{1.1}$ will be the resulting information package of the relationship.

$\sum P_{i}=1$

That $\mathrm{Pi}$ is assigned for any event taking place is the algorithm of information doing its work. It assigns probabilities from particle behavior, to size of galaxies to the tossing of a coin. If it were a computer program it would be the most complex computer program, but it's just existence, it is the computer program copying reality not the other way around. Humans are mimicking nature, that's what all human inventions can be because they come from information. $P_{i}$ is not consistent, it depends on location, many different variables can vary from location to location, conditions in a location change often. Given figure one, $\mathrm{P}_{1}$, the probability that $\mathrm{Xi}$ will have a relationship with $X_{1}$ and we get $X_{1 . i}$ varies from location to location depending on the variables in that location. This variation can be shown in the relationships

$P_{i}=t_{i}$

Such that $t_{i}$

$0 \leq t_{i} \leq 1$

Ti varies meaning that $\mathrm{Pi}$ varies according to the conditions, such that it therefore can be as low as having no chance of having $100 \%$ chance. The reason for this is to include location in the probability function, covering everything that can affect the probability of that event, relationship occurring. There is always the chance that a relationship that could not have occurred because of the location of information suddenly has the chance of occurring. The larger concept would catch it but the smaller concept of the algorithm of information around smaller and smaller locations would miss and thus it would seem of all a sudden an event can occur. But in the largest of concepts that probability was included a long time ago.

Thus, ti only affects Pi when specialization occurs, the probability of a specific event occurring will always be changing. The destiny of the larger location is more set depending on the relationship of the smaller location to the larger location, the relationship of the smaller information package to the larger information package also determines the probability of events, affects ti. Looking at figure 1, we understand that an information package has the chances of it entering a relationship determined by a probability function that is more easily understood as an algorithm. That information package itself must have been part of an event that led to the relationship of its existence. This is illustrated in figure 2, how we get to information package, Xi. Note though, because information package exists, it's the probability of existence is $100 \%$, we are just looking at Xi just before it existed. 
Figure 2: Origins of $X_{i}$

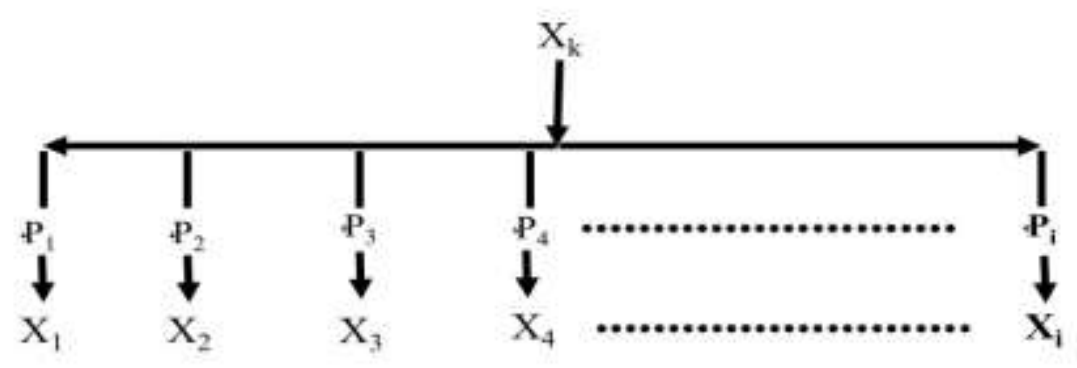

As can be seen from figure $2 \mathrm{Xi}$ comes from XK. The main purpose of figure 2 is that we appreciate everything is in a relationship and comes from a relationship. That everything is in a relationship and comes from a relationship explains complexity, in the future it would be important in school curricular, a 13-year-old should understand this, thus understanding whatever it is that they desire to look into in the future of their lives, it all has the same basic characteristics, it is all information made up of the same stuff.

Everything is information such that any information package $X$ ? will eventually be broken down to its elementary particles. The original thought around the algorithm of information begins here, a time period just after the big bang, following conventional thought of the time. It is said conventional thought of the time because events and time are accepted to have started with the big bang. Everything is accepted to have started with the big bang, a monumental event, started with the big bang or something similar. Thus, the original thought around this concept was based on the thoughts of our times. This should not be unusual, as we will see when we read further, though it is good to be with the herd, very often we miss the obvious for that emotional safety. Emotional because in a herd the intellectual is accepted emotionally, thus if one misses the obvious all miss the obvious because of the emotional attachment to not mentioning the obvious. Because there is a certain way of doing things. The only way of doing things is something that results in consistent patterns within information because all relationships are similar.

The original logic behind the algorithm of information was immediately after the big bang then it took effect determining all probabilities since. The idea being with that explosion, the particles came into existence and began having relationships all in a random manner. One being aware that each relationship having been a chance also comes with characteristics of that chance being successful, characteristics that will be specific to that relationship.

\subsection{Determining Existence}

Basic particle Ki has the probability of forming another relationship with another basic particle, as illustrated in figure 3.

Figure 3: Particle $K_{i}$ Probability of Relationships

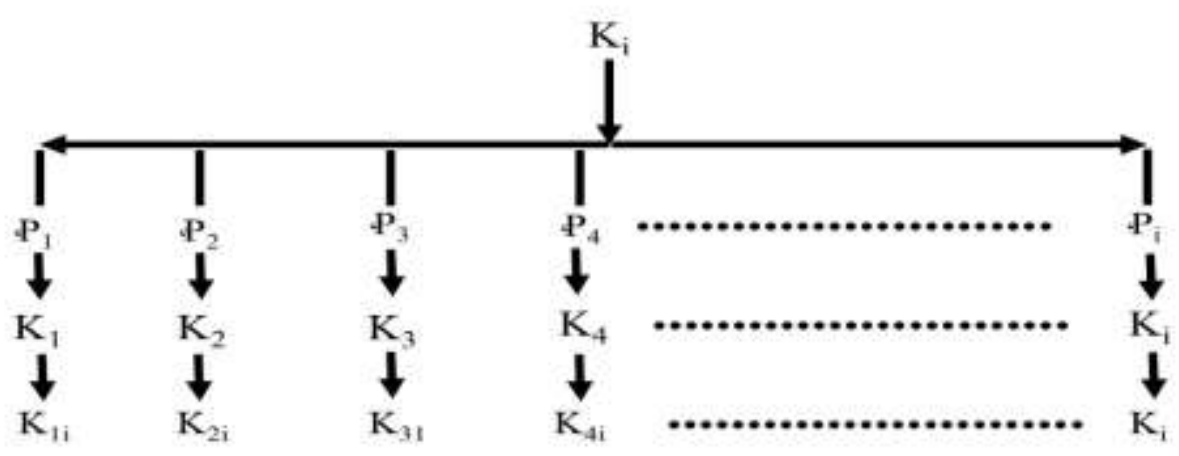


Figure 3, for example, shows that there is probability $\mathrm{P} 4$ of particle Ki having a relationship with particle K4 such that we get K4i. At times $\mathrm{P} 4$ can be zero or certain. It just sounds more appropriate to say a probability lies between 0 and 100 than $o$ and 1. Between 0 and 1 already implies that information does not need to be discrete, but psychologically between 0 and 100, there is less chance of using a decimal. When we talk of the probability of Ki having a relationship with $\mathrm{K} 4$ an important concept must be introduced, a concept that may underline the very principle of why information is discrete. It is wise to pay attention now; we are talking about the basic operations of this universe. Once a probability is assigned by a function, by the algorithm of information it means if, by chance that event occurs, it means a new relationship is created and that new relationships have specific characteristics with information being in that type of relationship. Hence a proton will behave as proton because of the underlying relationships of information that make up a proton.

The characteristics are part and parcel of the algorithm of information. If computer programmers understood this, in their attempts to mimic reality ever more precisely they would take note of this. These characteristics are at the beginning are the basis of classifying, categorizing, and identifying information. For every probability, there are characteristics assigned with that resulting relationship if the chances for that probability are greater than zero.

This is all fine, but if we accept that everything is in a relationship, one more question needs to be asked. Is the basic particle exempt from being in a relationship because it is the most basic piece of information we can have? If they are to be exempt from further scrutiny, what is the evidence to suggest this? Surely it can't be because they are called basic particles, a rose by any name would smell the same. The question we need to ask, if the particle is not exempt from relationships, then where does the particle come from, what event created the relationship that is a basic particle. Our first clue to answering this question is to understand that everything is information, and it is also made up of the same stuff. This everything includes stars, galaxies, life, asteroids, gold, hydrogen. This argument, this reality that everything is made up of the same stuff starts with the basic particle, everything is made up of basic particles. The basic particles themselves are differentiated.

Should not the reality that everything is made up of the same stuff be taken one step back. The algorithm of information is suitable for explaining every relationship, simply it is a random event. Once the event occurs it is set. There is no other way it could have happened, then the very laws of the universe must be different if that event did not occur. This is because once an event occurs, it was meant to occur no matter how improbable it seemed before it occurred. There is no time travel, one does not get to go back and change the results. This question of time travel can also be answered by asking where do particles come from? This requires taking the algorithm of information one step back. Doing this opens our thoughts that would be difficult to imagine without the idea of the algorithm of information.

The algorithm of information was thought to begin from the particle and up, being initiated at the time of the big bang. But basic particles are the information, it's how we understand that everything behaves in a similar manner. If everything is in a relationship, and those relationships lead to a loss of freedom, that loss of freedom being the energy needed to maintain that relationship and locked up with the specific characteristics that go with that relationship. Particles themselves are part of everything. What are the relationships that created particles, they can be classified, categorized, this is an electron, this a quark, this a photon, basic particles are not all the same? Asking where particles come from leads to understanding the roots of the algorithm of information.

That everything is made up of the same stuff must apply to the particle, meaning that particles are created by a relationship, they did not just appear. Before moving forward, it is important to understand what is being implied. We are going to the event called the big bang and in some instance's moments before the big bang. We can do this thanks to the neutrino. The argument rests on the principle that everything is made up of the same stuff that is why the basic characteristics of everything are the same. It is for this reason that the algorithm of information allows us to peak at a time before the big bang. It is best now to ask where do neutrinos come from? 
The importance of asking the question where neutrinos come from is because we know neutrinos can come from the properties of other particles as they enter relationship. Relationships between 2 protons in certain circumstances results in neutrinos being created. Relationships between protons and electrons also, in certain circumstances results in a neutrino being created.

The above paragraph leads us back to one of the major arguments why everything is information, it is all information in part because it is all made up of the same stuff. This takes the algorithm of information to before the particle. This would make sense even without the neutrino, but the neutrino confirms what is expected. Firstly, by the reality that our existence is bound by relationships, why should particles be exempt as if they are not part of our existence. Secondly, neutrinos, protons, and electrons must be made of the same stuff. There is no magical transformation when a neutrino is a result of some of these relationships, it is just a rearrangement of the same stuff. All particles must be made of the same stuff. That protons, electrons can be involved in relationships that create neutrinos is more than enough to accept the reality that everything is in a relationship, and these relationships lead to a loss of freedom. Understanding this allows us to see how basic particles are assigned their characters right before the beginning of the big bang is the beginning. A beginning with too many unanswered questions.

Besides evidence from the neutrino, there are experiments that have been carried out, but nobody was looking to answer the question where do particles come from, and how do they come to be? Note the simplicity of the process when understanding that everything is information and is in a relationship. Those who study basic particles without understanding this, cannot appreciate without being told that if the neutrino can come from protons and electrons, they must all be made up of the same stuff, right in front of our intellect.

Being made of the same stuff, the only phenomenon that physically differentiates basic particles is their mass. This is the beginning of the algorithm of information, to assign a probability and a characteristic for each particle. A process that could only have begun before the big bang, this shall be further investigated in this paper. There are 2 relationships representing 2 qualities of information must first be understood. These are illustrated in Figures 4 and 5.

Figure 4: Mass Particle Relationship

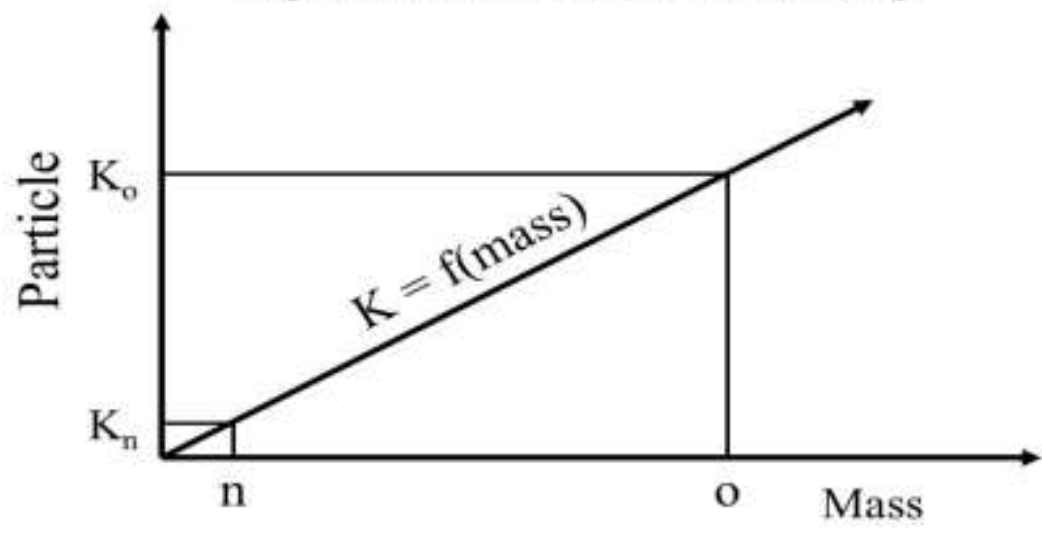

Figure 4 illustrates the relationship between a particle and the mass. The function for illustration's sake is simply named $K=f$ (mass). One can clearly see the limits of the function lie between $n$ and o such that the function $K$ $=f($ mass $)$ is limited such that:

$\mathrm{n} \leq \mathrm{K}=\mathrm{f}($ mass $) \leq \mathrm{O}$

This means a particle can have a minimum mass of $n$ and a maximum mass of $o$. Thus figure 4 illustrates the limits of the particle. 


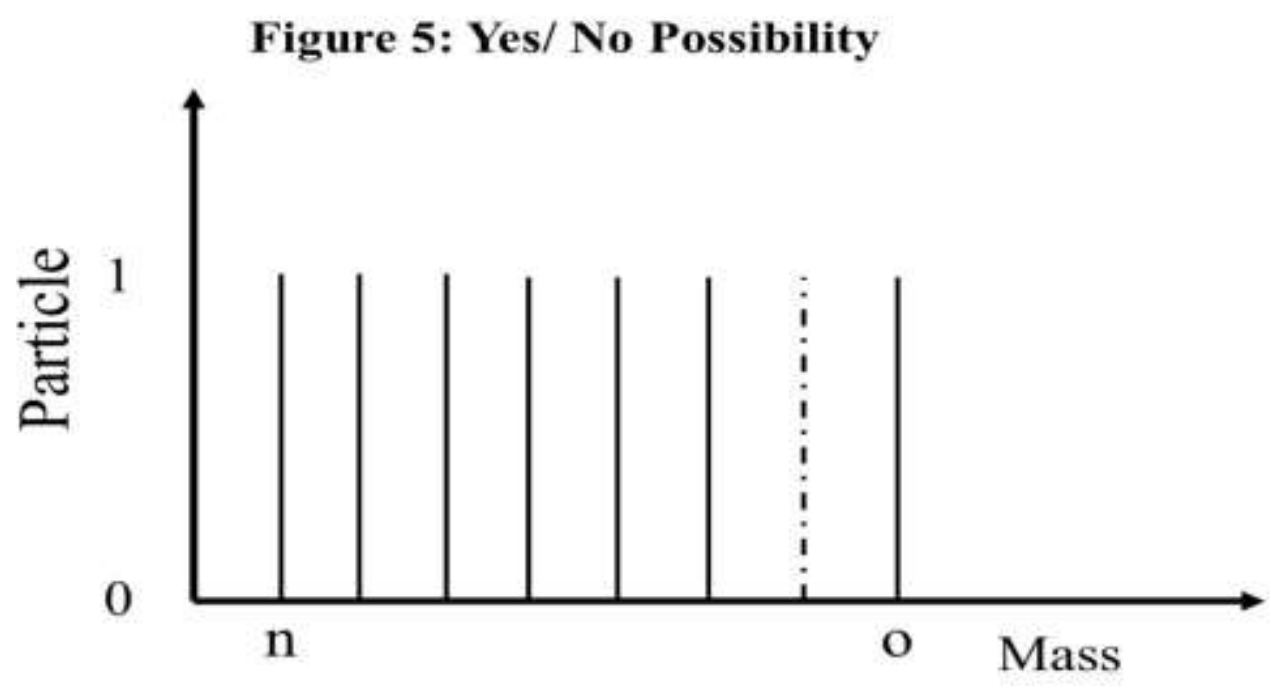

Figure 5 shows a Yes, No possibility. Yes, it means there is a chance of an event happening. This is represented as 1 and) in figure 5. The 1 means that the probability of the event is greater than 0 , and the 0 means there is no chance of event. The mass starts at $\mathrm{n}$ and ends at $\mathrm{o}$. Figure 5 illustrates that there will be classes of particles, but not all the mass range becomes particles. Having a chance of becoming a particle depends on having the correct mass, this correct mass is determined by the algorithm of information.

Understanding figure 4 and 5, we can get to the original algorithm of information that could easily be proved by a diligent person who believes in other things beyond relationships. But results of experimentation should fulfill even the most skeptic. This leads to the original algorithm of information, not after the particles where created as was originally envisioned, but by taking it one step back, understanding that all particles are made of the same stuff, take it one step back, to where did the particles come from.

\section{Figure 6: Beginnings of Algorithm of Information}

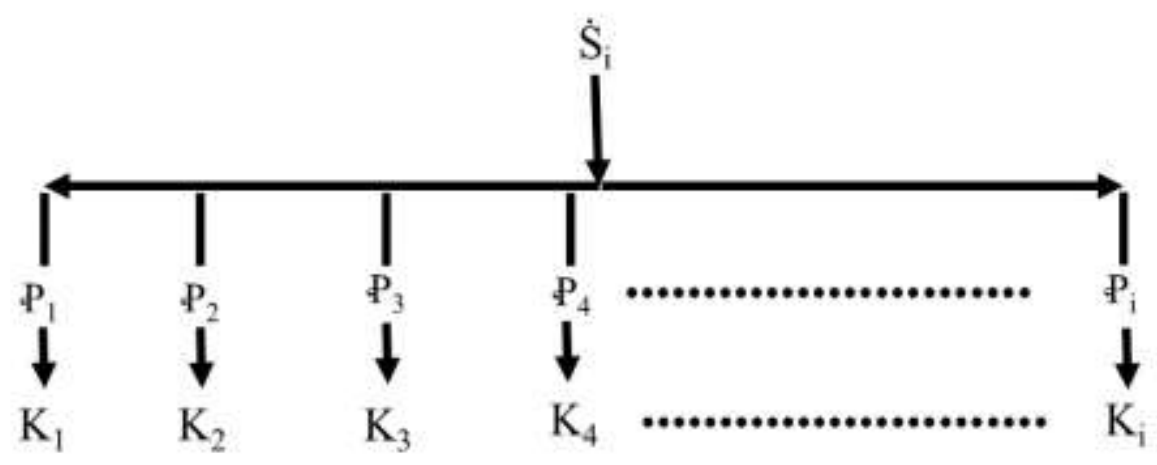

Figure 6 an illustration of the original action of the algorithm of information. Everything is made up of the same stuff, the neutrino gives us insights into this, and further collecting of experimental results will prove this. For now, let us take Si as some phenomenon, we just want now to understand original events. Si has no mass, given the right conditions it can have mass. The mass it can gain is defined by figure 4 , it is set between $n$ and $o$. Thus, it gains any mass if it is in certain parameters. Appreciating figure 5, we understand that there is only a chance to take certain mass, anything else will not work. Thus, looking at figure 6 , there is a probability $P 4$ of getting particle K4. It is not difficult to be accurate. Let us say that P4 is the probability that we get a quark, K4. We can actually calculate this probability by counting the amount of quarks in the universe, this is done by calculating 
the number of protons and neutrons and multiplying it by 3, multiplying that with their mass and we can get a figure over the total mass of the universe, and we would get the relationship:

(\# of quarks $x$ mass of quark) / Mass of universe

From protons and neutrons, we can also get the number of gluons and knowing their mass get

(\# of gluons $x$ mass of gluon) / mass of Universe

In general, thus we can say the algorithm of information assigned a probability to a particle existing at the beginning by:

(\# of particle $\mathrm{K}_{\mathrm{i}} \times$ mass of particle $\mathrm{K}_{\mathrm{i}}$ )/ mass of Universe $=\mathrm{Pi}$

From (7) we get (8):

$\sum$ (\# of particle $\mathrm{K}_{\mathrm{i}} \mathrm{x}$ mass of particle $\left.\mathrm{K}_{\mathrm{i}}\right) /$ mass of universe $=1$

The original mass is unique, it was determined by the amount of stuff that could become mass given the conditions when the universe came to be. The universe today still has the same amount of mass as it did when it begun 14 billion years ago. Knowing this, we can accurately calculate the original probabilities of particles, how they came to be by merely respecting equation (7). The meaning behind equation (7) no matter how refined defines the first event, defining the first events is beautiful enough, but to describe the first event, there is nothing spontaneous in this universe, everything is triggered determined by a random function, it is in a relationship or changing relationships.

We do not detect for example quarks, gluons flying loose around the universe. There was a set amount of potential mass, a critical amount of potential mass that would set the conditions that would lead to the big bang. This is in such a way that the algorithm of information seems deterministic and seemingly not random. The key word here is potential mass, not matter. The universe can only be from a potential if the physics has been correct since Newton, and we can assume it is correct because we have everything is in a relationship, that must come from somewhere. The big bang was a result of relationships, why did it not happen before, why did it not happen after, keyword being happened, when it happened conditions were just right, these conditions had to be before the big. Thus because of the keywords massed and happened we realize it was a probability function, why did it happen 14 billion years ago not 223 billion years ago, why not 1 billion years ago. It means the conditions where just right 14 billion years ago, and that can only mean pre-black hole relationships. By looking at it from relationships, understanding that particles are made of the same stuff, conditions where just right for formation of mass, the big bang is about celebration. The algorithm of information explains how this mass came to be. Mass does not matter. The big bang was a random event, it is just looking from hindsight it looks like it had to happen, no, it is as random as the time one work up this morning if they are lucky to have woke up at all. Though looking deterministic, the algorithm of information from the beginning was random, that is why the relationships where just right to cause events to happen 14 billion years ago, not 128 billion years ago or 200 million years ago. All a fantastic chance of relationships that are random events.

When we look at equation (8) and remembering that probability functions are always changing, the explanations for equations (2) and (3) explain this. After the event probabilities are always changing, neutrinos are continuously being created in stars, a balance that was not their pre-big bang. The algorithm of information at pre bang just determined how much mass was needed for that event to take place, and it was achieved by a random event. There are more neutrinos today than 12 billion years ago, stars are constantly creating neutrinos, stars are constantly emitting photons, but the mass of the universe has not increased though the variation of particles has greatly changed. 
The start was determined by the right conditions, a probability function set by the algorithm of information. We know the conditions where right and all the mass had a function such that every quark, matched every gluon, matched every electron such that thus far we have not seen gluons traveling the universe like photons and neutrinos. Given that every relationship is random and limited and determined by a probability function such that if we could work our way back using the algorithm of information, we could know exactly how many particles of each type where there 0.1 seconds after the "big bang" already been done in many aspects. But the algorithm also makes us understand that we could even track the origins of a proton in one's teeth and all the relationships it has been through back to 0.1 seconds after the big bang, and even the parts that went into before the big bang. Yes, there is a camp that is suggesting that the universe has always existed, and there is no need for a big bang or something similar. This is denying what we are looking at let alone mathematics. Our sun is spewing a lot of photons every second, it is getting smaller every second. Given that all the particles are made of the same stuff, that stuff had to be divided into these particles, that had to happen before it was always here. To understand, an electron is made up of more of the same stuff than a neutrino.

We can see some sort of pattern.

- There is the algorithm of information

- Determines the mass of particles

- Determines how many particles and types of particles thus determine conditions of the right amount of mass there shall be so that there are no loose ends, not even one loose end out of trillions upon trillions, gazillions of particles.

- Big Bang as mass is introduced

\subsection{Speculations of Before Light}

In figure 6, the concept of Sंi was introduced, why? Why the notation, Si. We have matter, it attains mass, and this is done through a probability function, Si becomes a particle, and the particle it becomes is determined by a probability function. Anything outside the limits of this function will not be stable, and we can see this in colliders. A large variety of what is considered exotic particles are formed all from the same stuff, but they are unstable, thus quickly lose their mass. Only a certain mass can be stable. Si is a string, it can only be. This string must attain a certain mass to be stable and have a characteristic, the mass it can attain lies between $\mathrm{n}$ and $\mathrm{o}$ in figure 4. Si represents the end of quantum mechanics, there are no more particles, we are at the very beginning of the particle. The string is discrete because the particle is discrete, this is the sub-quantum. The string is involved in a relationship we get mass and particles. Thus, the reality of relationships remains at the pre-quantum stage, pre-big bang before let there be light.

\subsection{Time Travel}

If particles do travel back and forth in time it would have started at the beginning, it would have been a quality of information with milliseconds of the big bang. Where would the particles be going, the time has just started, do they go back to conditions they could possibly not exist. How far back in time does a particle go back. If 5 seconds, 5 seconds before the big bang is another concept all together with no particles in existence, the conditions for particles to exist being impossible, there is no mass. Nothing can go back to conditions it could not exist, to its own nonexistence.

\subsection{The Experiment}

Luckily for us in large part the experiments have already carried out. It is carried out every time particles collide in a collider, the more influential the collider, the better. There are a variety of exotic particles that come out of smashing say neutrons together, they are all made of the same stuff, all coming out of smashed neutrons. 
Colliders were built to smash particles to get to understand the particle. If we had understood that everything is in a relationship when colliders where at proposal stage, a simple proposal title would have been, need for a collider to prove everything is made up of the same stuff. With the first collusion if anybody had bothered to look at everything would have been proved to be made of the same stuff, including the particles.

Anything that has ever come out of a collider, mostly unstable particles are made up of the same stuff. Stability is determined by the algorithm of information. For example, a neutrino has less than a Higgs boson but is stable, it just doesn't lose its mass. Coming from everything is in a relationship and understanding that everything is made up of the same stuff, the Higgs Boson is no more a foreign particle than all the other unstable particles. Understanding that things are relationships has some advantages, stability and unstable particles are all about relationships, and the properties of those relationships.

\subsection{Further Realities}

It must never be forgotten that the "big bang" was a probability, we know because we know when it occurred, why didn't happen earlier or later. To understand what is about to be said, we must understand what is going on in a collider, mass is being destroyed. For each particle destroyed, the universe has less mass.

Being a random event means that conditions, where right to occur, but the stuff that makes you the particles are still out there not all of it, was used up. When we smash particles together, we add to this stuff, as the unstable material becomes this stuff. That means if we understood the process, the relationships, we could theoretically add particle $\mathrm{Ki}$, adding mass to the universe. Philosophically this reality is in line with the ideas from the photonic cycle. A possible reason for the universe to create life. The relationship of life to the rest of the information.

But we must also always question ourselves, is it to correct to say everything is in a relationship and everything can be 10 isolated at the same time. Thus, speaking from purely a scientific viewpoint nothing can be isolated. It would rather we say we can categorize all information through observation, through being informed of what a thing is. If you think about it seriously, I made the mistake of not thinking it through, when somebody shows you it looks like a silly mistake, I should have known better, but I didn't. Even a prisoner in the isolation wing is not isolated. They have a relationship with the walls, with the air they breathe, with the bed they sleep in, the chair they sit on, we can't escape relationships, isolation is nonexistence.

Understanding that it can only be categorization comes from having a discussion with 11Suzanne Ngatai, small discussion but very impactful. Thus, the basic characteristics of everything become:

- discrete

- independent

- $\quad$ can be categorized

- can be used to compute

- $\quad$ consistent (why it is important to understand logic)

- random

- $\quad$ Everything is in a Relationship 


\section{Conclusions}

Everything is made up of the same stuff, and it is in a relationship, and these relationships are determined by the algorithm of information. The algorithm of information just does not give the probability, the chances of a relationship but also the properties of that relationship.

We can see basic function that determines a particle. The particle must be limited in quantity of mass between $\mathrm{n}$ and $\mathrm{o}$. And even then, only certain mass can become particles. The characteristics of particles are determined by the mass of the particle. From experiments in colliders, just nobody was interested in the implications, we know everything is made up of the same stuff. All the particles can be categorized as stable and unstable particles, mass. Colliders produce a large variety of unstable particles some call exotic particles. From the viewpoint of relationships, the higgs boson is no more important than all the other myriad of unstable particles, why? One could very well argue that the most important particle, the one that gives all mass is the smallest particle possible, since everything is made up of the same stuff, it would have to be built around the smallest particle possible, and it would be unstable, less mass than light, but, it still has mass and thus can't go faster than light. The keyword is it would be unpredictable; it will not be going very far. All known mass can be divided by it, this smallest particle without a remainder. A characteristic that the higgs boson cannot claim to have, it is heavier than a neutrino, heavier than the photon, but both the neutrino and photon can be categorized as stable particles, so in what way does the presence of a higgs boson benefit a photon except to confirm that all particles are made of the same stuff.

In a collider, we see the destruction of mass, not by the antiparticle, but by smashing particles together resulting in mostly unstable particles. Thus, the smashing of neutrons together results in unstable particles resulting them in returning to massless matter. It is here we understand what happens when an antiparticle meets a particle, everything just goes to this everything that makes up all particles. Just that with the antiparticle it is immediate whilst with smashing neutrons together we first get unstable particles then it's gone to this everything. One is allowed tiny bits of intuition, surely understanding the differences between these processes that lead to same thing is the key to reversing the process and adding matter. We use the energy of the collider to add energy to that massless matter somehow and there, a big bang right there at CERN because the big bang mass arrived and was not destroyed as use of colliders is all about. Think about the above paragraph, what is obviously a reality, mass is being destroyed, limiting $E=M C 2$, to the world of particles, it does not enter what makes particles, relationship remains vital to understanding the process. Energy now is determined by how much of this stuff that makes up everything is needed to create mass, there is no mass that is enough. Energy is determined by the qualities of $\mathrm{S}$, before the big bang, before the particle. Energy becomes a function of $\dot{S}$ and this everything that everything is made of.

Understanding relationships simplifies a lot of the thinking process. Particles all originate from the same material, stable or not, that material we will call a string. Just for consistency's sake, because some people have postulated about the idea. We do not know the nature of this string, but its existence is now mostly assured because everything is made of the same stuff, such that had to be a time before the particle, particles had to be made of the same thing we have called konke, meaning everything in Nguni languages. The string is needed to contain this konke and give us particles.

Thus, we know that $E=m c^{2}$ is redundant here, if we want can be certain that

$E=f(\dot{S}, k)$

The limits of Einstein must be reconsidered when there is no mass, a massless reality. There is no infinite mass to be gained. Gravity is associated with mass, there is no mass in a massless reality. Before the big bang, there is no mass, if one, however, decides to believe in $E=m c 2$, their equations will not make sense and will thus have to compensate for these anomalies by adding what could be considered dummy variables to smoothen things out, to make sense of things. Dummy variables are a term from econometrics, tells you a lot. This always happens in science. The earth as the center of the universe produced a great time for those who loved mathematics, 
adding this and that to compensate for the fact that it does not make sense for the earth to be the center of the universe. $\mathrm{E}=\mathrm{mc}^{2}$ is not the center everywhere, it must be taken out then it does not need to be compensated for, one might find themselves losing many dimensions in their models. If one includes $E=m c^{2}$ and its principles not even the most powerful computer can solve the problem, because it will have to compensate for something that should not be there. Though we do not know nature of this string we can speculate, its very nature determines the reality of this universe at the most fundamental level. If all the string that contains konke are of the same size, that means that it can stretch to incredible levels.

Or is it strings within strings blurring concept of konke with strings? When we smash particles in a collider they break up, the particles break up. If the string contains konke and we get mass, that mass, all those exotic particles must also be contained by strings that fail to gather the required mass with enough characteristics to be stable. But it doesn't necessarily mean many strings. Strings can just form from another string; it is possible gluons, and photons show this characteristic as stable particles. Particularly photons, if we can take a photo of a photon it is self-replicating, if we can take many photos then it is verified as explained in the paper 9the Photon and endless energy. The mere act of taking a photo of a photon should be impossible unless it is self-replicating. That could be a possible behaviour of strings.

It is the interaction within konke, what unstable particles return to, we have named it konke, and that is that. This konke interacts with a string and we get mass, stable particles, all a probability function. Given the idea of fields, do we have different strings for each field, because somehow things have balanced? We need to relook at the concept of the field. If fields yield stable particles, they must be random events. One can clearly understand now that everything with the same mass has equal energy, all photons have the same amount of energy.

When we appreciate what is going on in a collider, we begin to understand an important concept about mass, that of potential mass. We can understand that when neutrons collide a lot of stuff becomes unstable and returns to what it was before it was neutron, with have named this stuff konke, and everything is made of this stuff. By losing mass in colliders and seeing these neutrons returning to this massless konke, we realize that the potential for this neutron is there, in this konke, such that there is potential for the neutron there in the konke. Thus, we can talk of infinite potential mass. No matter how much information was involved at the big bang, there is potential for much more information to have acquired mass in that random event. Relationships from galaxies to strings, pre-quantum, pre-big bang. We are getting closer to the source.

Thus, the greatest human scientific achievement possible understanding that everything is about relationships is to be able to pluck out a particle from konke, seemingly nothing. Unite those particles and create an atom, unite those atoms and create elements, unite those elements and create molecules, unite those molecules and create a cell. Unite those cells and create a multi-celled organism, you know where it goes from there, then we have totally mimicked the universe our teacher.

\section{References}

1. Khumalo, B. (2016) What is a Theory of Everything https://instituteofknowledge.blogspot.com/2016/10/what-is-theory-of-everything.html

2. Khumalo, B. (2016) A Treatise on Information, Knowledge, Economics and Technology http://www.universitypublications.net/ijbms/0502/html/T6N690.xml

3. Doeleman, S. (2019) Focus on the First Event Horizon Telescope Results https://iopscience.iop.org/journal/2041-8205/page/Focus on EHT

4. Khumalo, B. (2007) Point $X$ and the Economics of Knowledge https://ideas.repec.org/p/pra/mprapa/3735.html 
5. Khumalo, B. (2015) Information Theory and Dimensions: Enhancing Quantum Mechanics https://cirworld.com/index.php/jap/article/view/1352

6. Khumalo, B. (2016) The Theory of Everything: Newton, Information, Complexity, and Evolution, https://instituteofknowledge.blogspot.com/2016/11/the-theory-of-everything-newton.html

7. Khumalo, B. (2017) Knowledge Economics: Summary and Rationality https://www.scirp.org/journal/paperinformation.aspx?paperid $=81147$

8. Castro, B, J. (2013) What are neutrinos, and how do they come from beyond our solar system? https://io9.gizmodo.com/what-are-neutrinos-and-how-do-they-come-from-beyond-ou-513336033

9. Khumalo, B. (2017) The Photon and Endless Energy https://www.scirp.org/journal/paperinformation.aspx?paperid=78495

10. Geneste, J F. (2019) question period after the presentation (https://www.youtube.com/watch?v=PyGvVM94EUo\&t=8s), how can anything in existence be isolated? https://www.scientificfederation.com/nucleaur-engineering-2019/index.php\#about

11. Khumalo, B (2016) The Theory of Everything: Newton, Information, Complexity, and Evolution, https://instituteofknowledge.blogspot.com/2016/11/the-theory-of-everything-newton.html

\section{Thanks}

Thanks to the Scientific Federation for Inviting me to a conference, having nothing to present, I wrote this paper. As well as for the conference discounts 